\title{
Outward-inward information flux in an opinion formation model on different topologies
}

\author{
A.O. Sousa ${ }^{1}$ \\ Institute for Computer Physics (ICP), University of Stuttgart, \\ Pfaffenwaldring 27, 70569 Stuttgart, Germany. \\ J. R. Sánchez ${ }^{2}$ \\ Fac. Ingeniería - Univ. Nac. de Mar del Plata \\ Justo 4302 - 7600 Mar del Plata - Argentina.
}

\begin{abstract}
A simple model of opinion formation dynamics in which binary-state agents make up their opinions due to the influence of agents in a local neighborhood is studied using different network topologies. Each agent uses two different strategies, the Sznajd rule with a probability $q$ and the Galam majority rule (without inertia) otherwise; being $q$ a parameter of the system. Initially, the binary-state agents may have opinions (at random) against or in favor about a certain topic. The time evolution of the system is studied using different network topologies, starting from different initial opinion densities. A transition from consensus in one opinion to the other is found at the same percentage of initial distribution no matter which type of network is used or which opinion formation rule is used.
\end{abstract}

Key words: Social systems, Opinion formation, Structures and organization in complex systems, Computer modeling and simulation.

PACS: 89.65.-s, 89.75.-k, 05.10.-a

\section{Introduction}

Recently, there has been a growing interest in the study of complex phenomena appearing in heterogeneous areas different from the traditional fields of

1 E-mail address: sousa@ica1.uni-stuttgart.de, aosousa@gmail.com

2 E-mail address: jsanchez@fi.mdp.edu.ar 
research. In particular, one of those interesting fields turns to be the application of statistical physics methods to social phenomena. At present discussions of such heterogeneous aspects can be found in several reviews $[1,2,4]$. One of the sociological problems that attracts much attention is the construction of consensus out of some initial condition and different models have been introduced in order to simulate and analyze the dynamics of opinion formation processes $[2,3,4,5,6,7,8,9,10,11,12,13]$.

Mainly, opinion formation models are composed by interacting agents usually represented by the state adopted by certain variables. Agents interact between each other forming a network with a certain topology. Models differ to each other in three aspects: the type of variables used to represent the opinion of the agent, the type of interaction imposed between the agents and the topology adopted.

Here, in order to maintain certain degree of generality, the dynamics of consensus formation is studied using different topologies. Each agent is represented by a spin state variable representing the opinion in favor $(+1)$ or against $(-1)$ about a certain topic and the interaction between the agents is randomly changed during the evolution between two possible mechanisms, the Galam majority rule [2] and the Sznajd updating rule [5]. This last model is a successful Ising spin system describing a simple mechanism of making up decisions in a closed community in which the opinion influence flows outward a given group to the nearest individuals. In the Sznajd model a pair of nearest neighbors convinces its neighbors to adopt the pair opinion if and only if both members of the pair have the same opinion; otherwise the pair and its neighbors do not change opinion. The Sznajd consensus model has rapidly acquired importance in the new field of computational socio-physics $[1,4,5,6,13,14,15,16,17,18,19,20,21]$.

Additionally, it is meaningful to mention that consensus models are set up in complex networks with different topologies. The statistical properties of realworld social networks vary strongly, for example, the degree distribution can be single-scale, broad-scale or scale-free [22]. Due to the lack of a single model covering the topological features of social networks, we consider a few well established network models aiming to unveil the effect to different aspects of the topology:

- standard one- and two- dimensional regular lattices;

- Watts-Strogatz small-world network [23];

- Erdős-Rényi random graph network [24];

- Triad scale-free network (Barabási-Albert-like model) [25,26]. 


\section{The Model}

On the system lattice, each site $i(i=1,2, \cdots, N$; where $N$ is the total number of sites-agents) carries a spin $s_{i}$, which has two possible directions, $s_{i}=+1$ or $s_{i}=-1$. The state of these spin-like variables can be considered to represent an agent or individual carryng one of two possible opinions, against or in favor about a certain topic: $s_{i}=+1$ represents a positive opinion and $s_{i}=-1$, a negative one. Initially the opinions are distributed randomly, $s_{i}=+1$ with probability $p$ or $s_{i}=-1$, otherwise.

\subsection{Opinion updating rules}

As stated above, during the time evolution of the model, each agent can use two different strategies in order to update its opinion state variable $s_{i}^{t}$,

- with probability $q$ the agent opinion is updated according to a kind of Sznajd rule $[4,5,9,16,18,19]$ :

- square lattice and random graphs: 2 sites convincing rule: For each site $i$ chosen, we select randomly one of its neighbors. If this selected neighbor has the same opinion as the site $i$, then all their neighbors follow the pair's opinion. Otherwise, nothing is done.

- usual 1d regular chain: Two updating rules have been applied: a) the original Sznajd rule [5]: $s_{i-1}^{t}=s_{i+1}^{t}$ and $s_{i+2}^{t}=s_{i}^{t}$; b) 2 sites convincing rule.

- with probability $1-q$ the agent opinion is updated using a Galam-like majority rule without inertia in which individuals gather during their social life in meeting cells of different sizes where they discuss about a topic until a final decision, in favor or against, is taken by the entire group.

$$
s_{i}^{t}=\operatorname{sign}\left(s_{i}^{t}+\frac{\sum_{j \in \wedge_{i}} s_{j}^{t}}{k(i)}\right),
$$

where the $\operatorname{sign}($.$) function takes the value +1$ if the argument is positive and the value $-1, k(i)$ is the number of vertices in the neighborhood $\wedge_{i}$ of site $i$ : the nearest neighbors in the usual one- and two- dimensional regular lattices and all nodes connected to $i$ in the random graphs. In the regular lattices there is never a tie. Although the random graphs have been constructed considering a node connectivity to avoid such a tie, in the case it occurs, then the individual randomly assumes an opinion \pm 1 . 


\subsection{Network topologies}

In this subsection we briefly describe the different topologies used, in addition to the standard one- and two- dimensional lattices.

\subsubsection{Watts-Strogatz small-world network}

Social networks are far from being completely regular or completely random. For instance, it has been found that most real-life networks display some common characteristics the most important of which are called small-world effect and scale-free distribution. The recognition of small-world effect involves two factors: the clustering coefficient and the average shortest path length, a network is called a small-world network as long as it has small average shortest path length and large clustering coefficient $[23,27]$. One of the most well-known small-world models is Watts-Strogatz small-world network (WS model) [23], which can be constructed by the following algorithm: the initial network is a one-dimensional lattice of $N$ sites, with periodic boundary conditions (i.e., a ring), each site being connected to $m$ nearest neighbors. We choose a vertex and the edge that connects it to its nearest neighbor in a clockwise sense. With probability $p_{s}$, we reconnect this edge to a vertex chosen uniformly at random over the entire ring, with duplicate edges forbidden; otherwise we leave the edge in place. This process repeats until one lap is completed and proceeding outward to more distant neighbors after each lap, until each edge in the original lattice has been considered once.

\subsubsection{Erdős-Rényi random graph}

The construction of the Erdös-Rényi random graph [24] starts with a set of $N$ isolated vertices, then successive edges are randomly added with a probability $p_{a}$. In this way, the total number of edges is $m_{t}=p_{a} N(N-1) / 2$ and the average number of neighbors of a node (degree or connectivity) is $\bar{m}=p_{a}(N-1)$. In the limit $N \rightarrow \infty$, the mean number of bonds per site can be approximated by $p_{a} N$ and a Poissonian connectivity distribution is observed. In our simulations, the graph has been built in such way that each node has at least $m$ links, although the coordination number of any particular vertex can be bigger than $m$ links ( $m=4$ in our simulations).

\subsubsection{Barabási-Albert and Triad scale-free network}

The most fashionable network presenting both properties, scale-free and smallworld aspects, is the Barabási-Albert scale-free network [25]. Although the BA network has successfully explained the scale-free nature of many networks, a 
striking discrepancy between it and real networks is that the value of the clustering coefficient varies fast with the network size $N$ and for large systems is typically several orders of magnitude lower than found empirically and it vanishes in the thermodynamic limit [26]. In social networks, for instance, the clustering coefficient distribution $C(k)$ exhibits a power-law behavior, $C(k) \propto$ $k^{-\gamma}$, where $k$ is the node degree (number of neighbors) and $\gamma \approx 1$ (everyone in the network knows each other).
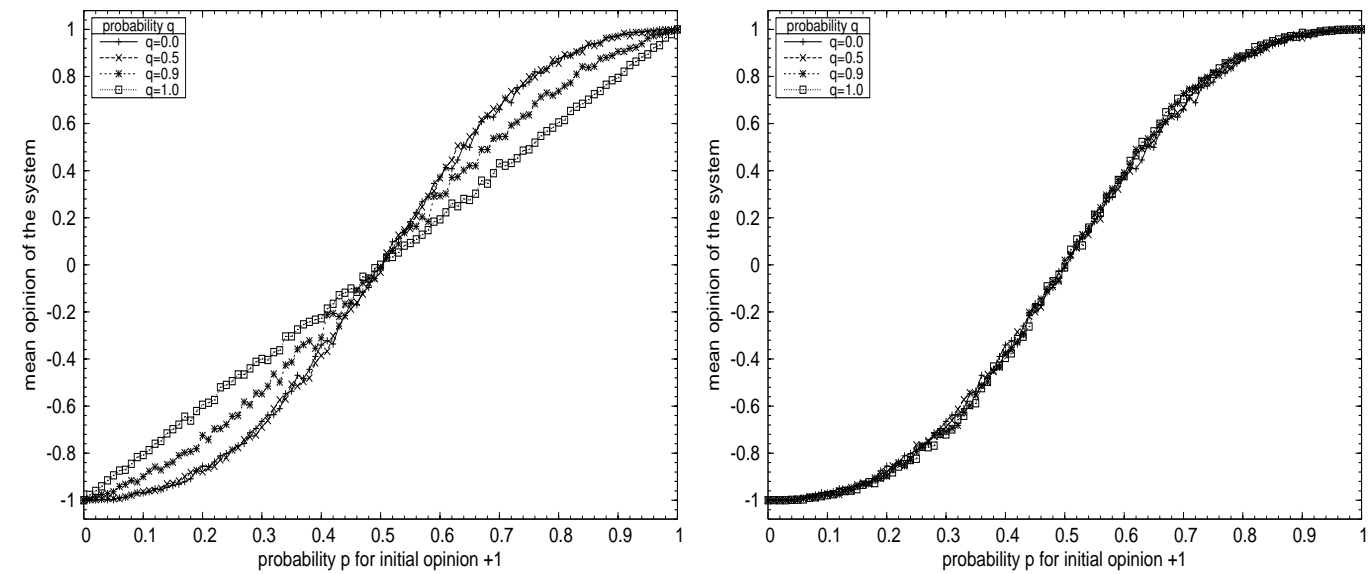

Fig. 1. The mean opinion density of the system as a function of the probability $p$ for initial opinion +1 for an one-dimensional lattice, different values of probability $q, N=500$ and $n_{s}=3000$. On the left, original updating rule, and on the right, 2 nodes convincing rule.
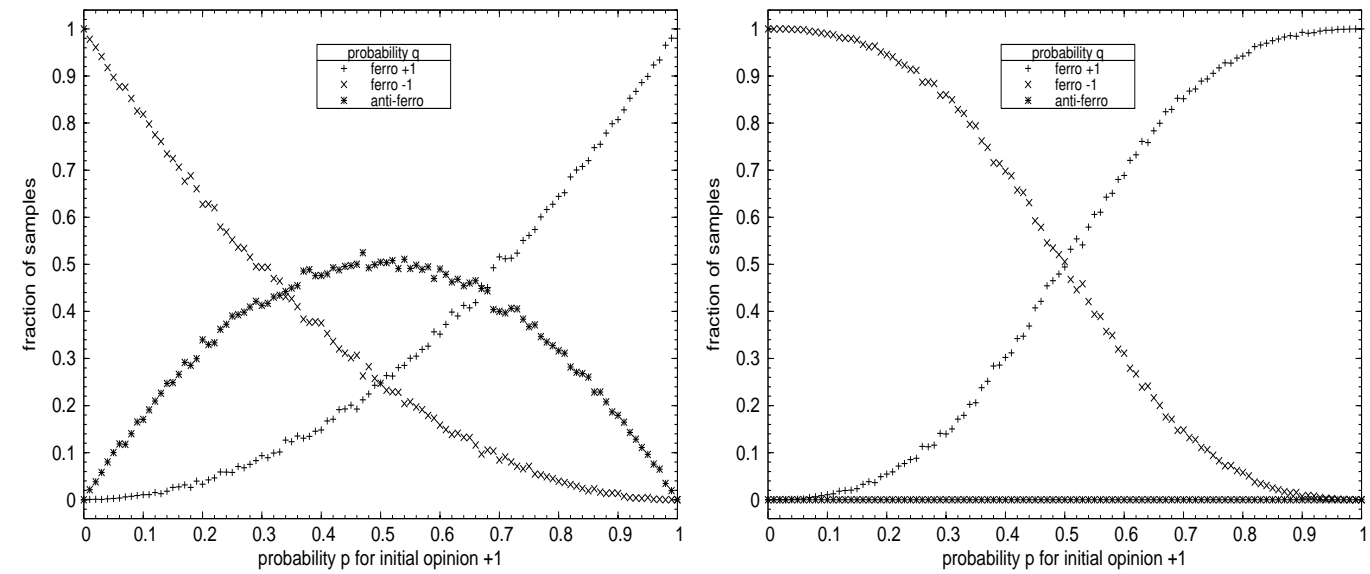

Fig. 2. Fraction of samples, out of 3000, which becomes ordered ferro-magnetically ( "all up" $(+)$ and "all down" $(\times)$ ) and anti-ferromagnetically $(*)$ as a function of the probability $p$ for initial opinion +1 for an one-dimensional lattice, $N=500$ and $q=1.0$. On the left, original updating rule, and on the right, 2 nodes convincing rule.

This problem has been surmounted and scale-free models with high clustering coefficient have been investigated, by adding a triad formation step on the Barabási-Albert prescription [26]. The Barabási-Albert network starts with 
a small number $m$ ( $m=4$ in our simulations) of sites (agents, people) all connected with each other. Then a large number $N-m$ of additional sites is added as follows: first, each new node (node $i$ ) performs a preferential attachment step, i.e, it is attached randomly to one of the existing nodes (node j) with a probability proportional to its degree; then follows a triad formation step with a probability $p_{t}$ : the new node $i$ selects at random a node in the neighborhood of the one linked to in the previous preferential attachment step (node $j$ ). If all neighbors of $j$ are already connected to $i$, then a preferential attachment step is performed ("friends of friends get friends"). In this model, the original Barabási-Albert network corresponds to the case of $p_{t}=0$. It is expected that a nonzero $p_{t}$ gives a finite nonzero clustering coefficient as $N$ is increased, while the clustering coefficient goes to zero when $p_{t}=0$ (the BA scale-free network model) [26]. Indeed, the clustering coefficient increases as the probability $p_{t}$ and $m$ increase.

\section{Results}

At every time step $t>0$, all the individuals $N$ are randomly visited and updated (a random list of nodes assures that each node is reached exactly once) by following the stated rules in Section 2.1. If a full consensus (all individuals have the same opinion) or if the maximum number $t_{\max }=100000$ of iterations is reached, the simulation ends. The curves presented here correspond to the results averaged over $n_{s}$ samples.

In Fig. 1 we present the mean opinion of the system as a function of the probability $p$ for the one dimensional model when different values of probability $q$ are considered. On the left side, the results are from using majority [2] and the original Sznajd [5] rules. As we can see, the mean opinion (or final magnetization $\left.\mathrm{m}_{\mathrm{f}}\right)$ is given by $\mathrm{m}_{\mathrm{f}}=2 \mathrm{p}-1$ when only the Sznajd rule $(q=1.0)$ is applied and as $q$ increases $m_{\mathrm{f}}$ quickly approaches a static value $\mathrm{m}_{\mathrm{f}}=\mathrm{m}_{\mathrm{o}}\left(3 /\left(2+\mathrm{m}_{\mathrm{o}}^{2}\right)\right)^{3 / 2}$, that depends only on the initial magnetization $m_{o}=2 p-1$. On the right side, it can be noticed that the system exhibits the same behavior for any value of probability $q$. Moreover, from this plot, we find that in both updating rules, the 2 nodes convincing and the majority rule, the system always evolves to a ferromagnetic consensus (either all up or all down) and that they provide the same probability distribution for the system to reach a fixed point with all spins up or all spins down (Fig.2, right), except in the anomalous case of an anti-ferromagnetic initial state $(+-+-+-)$. Nevertheless, when the system updates according to the original rule, besides the two ferromagnetic states, an anti-ferromagnetic fixed point is also reached (Fig.2, left). These results are in good agreement with previous studies on Sznajd, majority and minority models $[2,5,12,20]$. Very recently, a modification of the Sznajd model in order to avoid the unrealistic anti-ferromagnetic fixed point was proposed 
in Ref.[21].
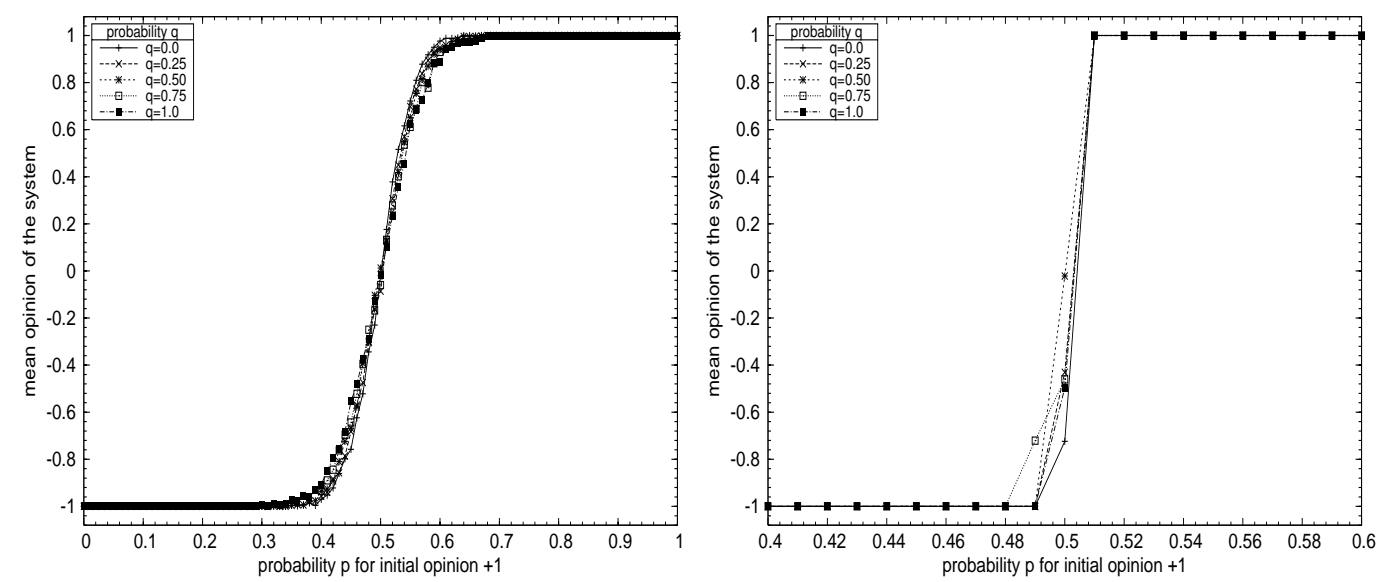

Fig. 3. As Fig. 1, but for the square lattice. On the left, $N=400$ and $n_{\mathrm{s}}=1000$, and on the right side, $N=490000$ and $n_{\mathrm{s}}=1$.

In Fig. 3, we observe the mean opinion density of the system as a function of the probability $p$ for initial opinion +1 for a square lattice, as well as for different values of probability $q$. From this figure, one can observe a phase transition: concentration $p>1 / 2$ lead to full consensus +1 and concentrations $p<1 / 2$ to full consensus -1 for large enough systems. Since in a finite network, of course, phase transitions are never sharp, and thus the transition is indicated numerically by a slope (Fig.3 left) becoming the steeper the larger the network size is (Fig.3 right). In an infinite network, one would get a sharp step function for the mean opinion of the systems versus the initial concentration $p$ of opinion +1 . Moreover, the results are the same independently of the value of the probability $q$, i.e., to update the system following Sznajd recipe or majority rule provides the same behavior and consensus threshold $p=1 / 2$.
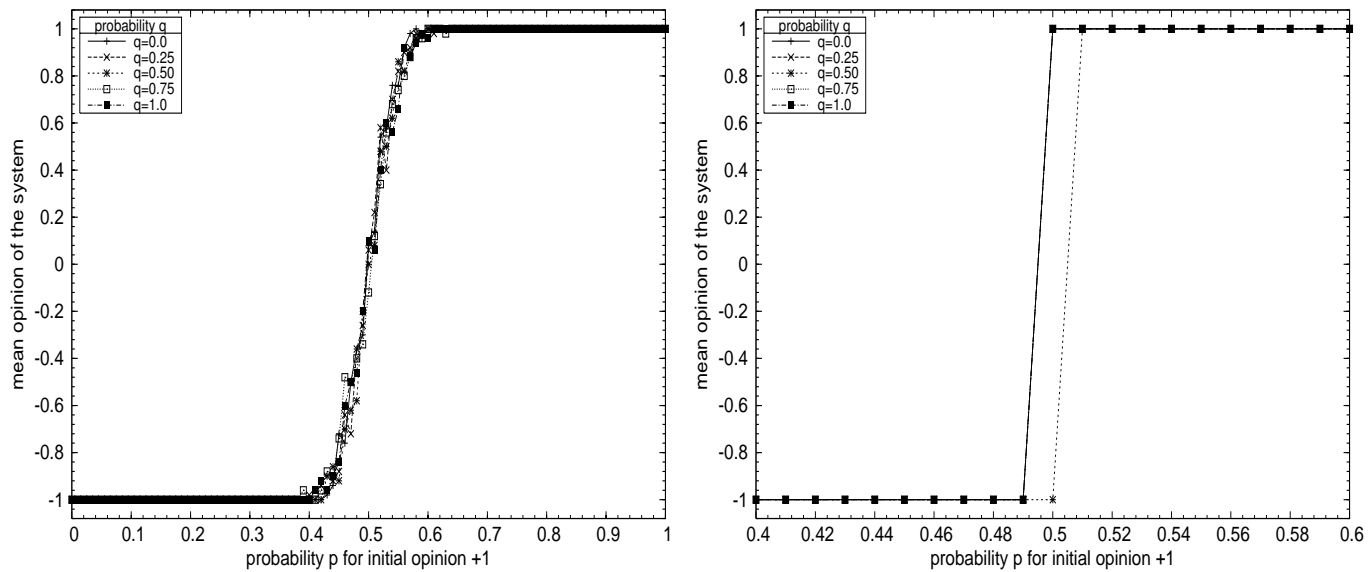

Fig. 4. As Fig. 1, but for Erdös-Rényi random graph. On the left, $N=500$ and $n_{\mathrm{s}}=100$, and on the right side, $N=500000$ and $n_{\mathrm{s}}=1$. 
Fig. 4 shows the mean opinion density of the system as a function of the probability $p$ for initial opinion +1 for the Erdős-Rényi random graph. As we can see, it presents the same pattern as in Fig. 3, so that the consensus threshold is $1 / 2$ and there is no difference related to the chosen dynamics for updating the system: Sznajd or majority rule.
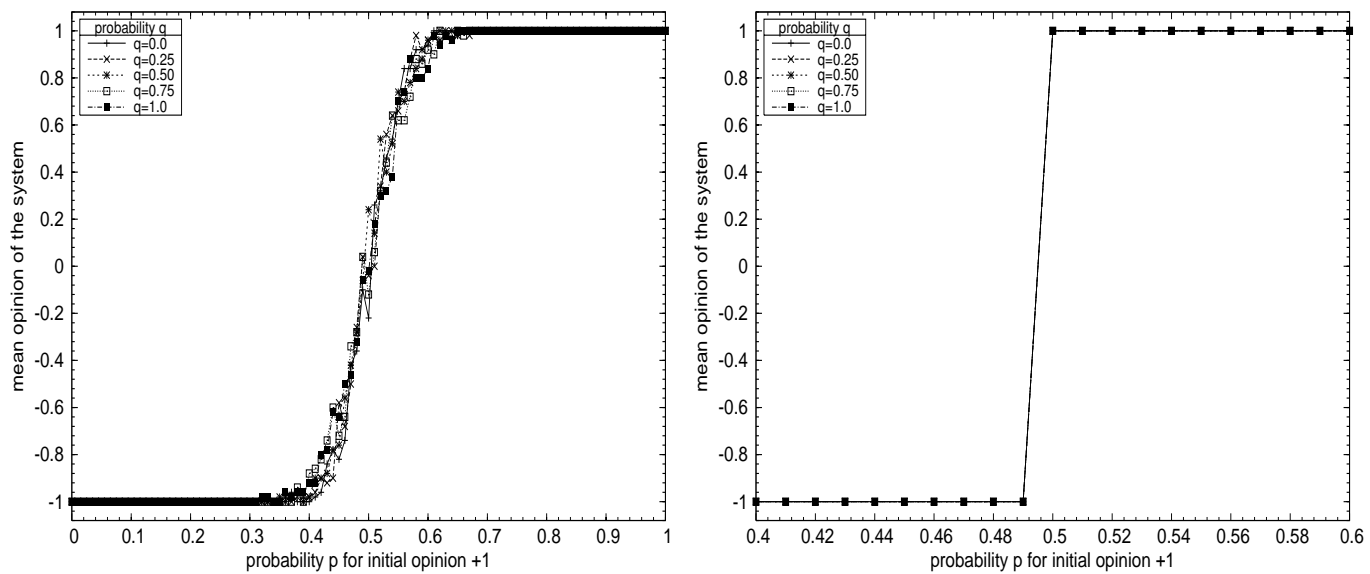

Fig. 5. As Fig. 1, but for Watts-Strogatz small-world network. On the left, $N=500$ and $n_{\mathrm{s}}=100$, and on the right side, $N=500000$ and $n_{\mathrm{s}}=1$. Others parameters: $p_{s}=0.1$ and $m=2$

In Fig. 5, we show the same as in Fig.3, but for the Watts-Strogatz small-world network. From this figure, it can be observed that the consensus threshold is also $1 / 2$ and the chosen updating dynamics does not exert any influence on the results, as already noticed for the square lattice and the Erdős-Rényi random graph.
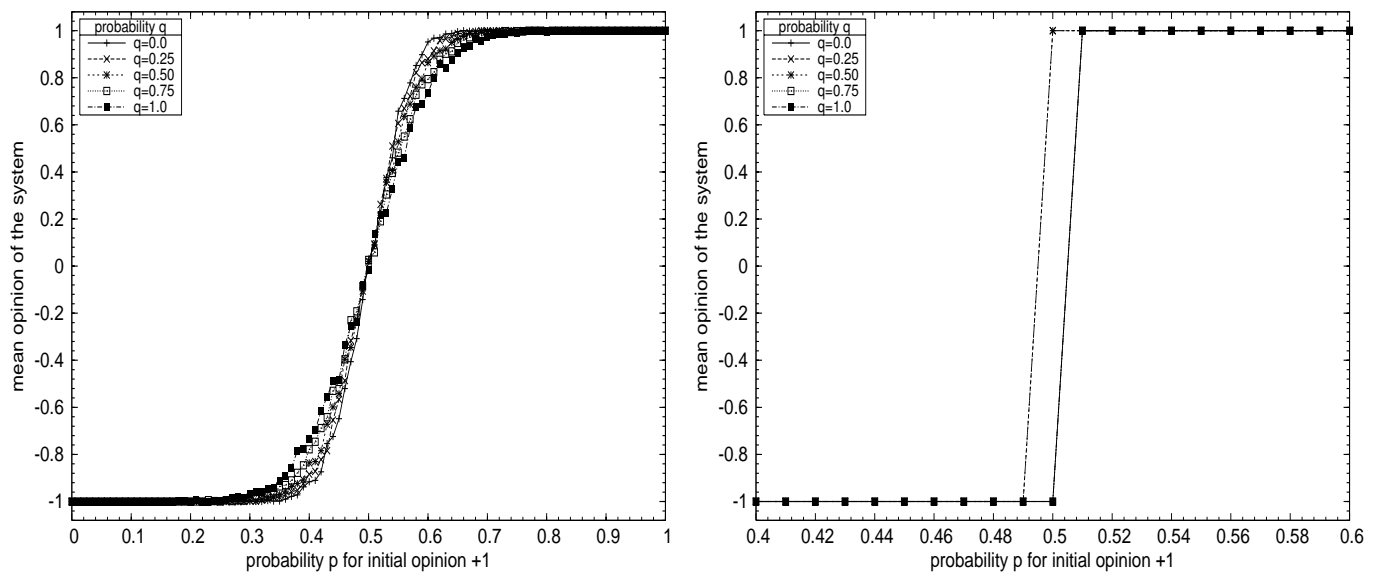

Fig. 6. As Fig. 1, but for the Triad scale-free network. On the left, $N=500$ and $n_{\mathrm{s}}=1000$, and on the right side, $N=500000$ and $n_{\mathrm{s}}=1 . p_{a}=m / N$, with $m=4$

Fig. 6 presents the mean opinion density of the system as a function of the probability $p$ for initial opinion +1 for the Triad scale-free network. The 
same consensus threshold $p=1 / 2$ and results completely independent of the adopted updating dynamics are once more observed.

The phase transition at $p_{c}=1 / 2$ here observed for the square lattice and for the random graphs does not exist in one dimension [5] or when a single site [15] (instead of a pair or plaquette) on the square lattice [9] convinces its neighbors, although it has been also found on the square lattice when a plaquette or a neighboring pair persuades its neighbors [9], on a correlateddiluted square lattice [14], on a triangular and simple cubic lattice if a pair convinces its 8 (or 10, respectively) neighbors [17], on the Barabási-Albert network [16], on a triad network [19] and on a pseudo-fractal network [18].

\section{Conclusion}

In this article, we have investigated a simple model of opinion formation in which binary-state agents evolve due to the influence of agents in a local neighborhood by two different updating rules: with probability $q$, the agents adopt the Sznajd rule and the majority rule with probability $1-p$. In one dimension, for $q>0$ the system always evolves to three fixed points: two of which refer to ferromagnetism ("all up" or "all down") and one to antiferromagnetism. As $q$ increases, the magnetization quickly approaches a fixed values that depends only on the initial magnetization. On the other hand, we have also obtained that the 2 nodes convincing rules corresponds to a kind of majority rule in which only a ferromagnetic consensus is observed with similar probability distribution for a consensus +1 or consensus -1 . Moreover, taking into account more realistic topologies, the interplay between majority and Sznajd rule leads the system to a phase transition as a function of the initial concentration of $S=+1$ opinions at $p_{c}=1 / 2$ : For $p<1 / 2$ all samples end up with $S=-1$, and for $p>1 / 2$ they all end up in the other fixed point $S=+1$, for large enough systems. Indeed, such consensus threshold $p_{c}$ of the opinion dynamics is not specific of the particular graph one uses to describe the social relationships between individuals, since it takes only one possible value $p_{c}=1 / 2$. Moreover, no matter whether the direction of the information flux is inward ( $q=0$, majority rule) or outward ( $q=1$, Sznajd rule) the system provides the same behavior. In fact, our results represent a natural outgrowth of recent works [2] on the interplay between the majority rule and Sznajd model which claim that the Sznajd model represents a particular case of a class of Galam majority model, that shows the same threshold value $p_{c}$. 


\section{Acknowledgements}

We thank D. Stauffer, S. Galam and K. Malarz for many helpful discussions, advice and a criticial reading of the manuscript.

\section{References}

[1] W. Weidlich, Sociodynamics - A systematic approach to mathematical modeling in social sciences, Taylor \& Francis, London (2002).

[2] S. Galam, Y. Gefen and Y. Shapir, Math. J. Socio. 9, 1 (1982); S. Galam, B. Chopard, A. Masselot and M. Droz, Eur. Phys. J. B 4, 529 (1998); S. Galam and J.D. Zucker, Physica A 287, 644 (2000); S. Galam, Eur. Phys. J. B 25, 403 (2002); S. Galam, Physica A 336, 49 (2004); S. Galam, cond-mat/0409484, A.O. Sousa, K. Malarz and S. Galam, cond-mat/0503702 (Int. J. Mod. Phys. C 16 (2005), in Press).

[3] G. Deffuant, D. Neau, F. Amblard and G. Weisbuch, Advances in Complex Systems 3, 87 (2000); D. Stauffer, A.O. Sousa and C. Schulze, J. Artif. Soc. Simul. (http://jasss.soc.surrey.ac.uk/7/3/7.html); A.O. Sousa, cond-mat/0406766

[4] D. Stauffer, Physica A 336, 1 (2004); D. Stauffer, Journal of Artificial Societies and Social Simulations 5, issue 1, paper 4(jasss.soc.surrey.ac.uk) (2002); Computing in Science and Engineering 5, 71 (May/June 2003); and in The Monte Carlo Method on the Physical Sciences, edited by J. E. Gubernatis, AIP Conference Proceedings, 690, 147 (2003) (cond-mat/0307133).

[5] K. Sznajd-Weron and J. Sznajd, Int. J. Mod. Phys. C 11, 1157 (2000).

[6] F. Slanina and H. Lavicka, Eur. Phys. J. B 35, 279 (2003).

[7] K. Malarz, Int. J. Mod. Phys. C 14, 561 (2003); T. Erez, M. Hohnisch, S. Solomon, Economics: Complex Windows, Eds. M. Salzano and A. Kirman, Springer, 201 (2005) cond-mat/0406369).

[8] P. Gawroński, K. Kułakowski, physics/0503085 P. Gawroński, P. Gronek, K. Kułakowski, physics/0501160 K. Kułakowski, P. Gawroński, P. Gronek, physics/0501073

[9] D. Stauffer, A.O. Sousa, and S. Moss de Oliveira, Int. J. Mod. Phys. C 11, 1239 (2000).

[10] D. Vilone, A. Vespignani and C. Castellano, Eur. Phys. J. B 30, 399 (2002).

[11] C. Castellano, M. Marsili and A. Vespignani, Phys. Rev. Lett. 85, 3536 (2000). 
[12] P.L. Krapivsky and S. Redner, Phys. Rev. Lett. 90, 238701 (2003); M. Mobilia, Phys. Rev. Lett.91, 028701 (2003); M. Mobilia and S. Redner, Phys. Rev.E 68, 046106 (2003).; D. Makowiec, Lecture Notes in Computer Science, Publisher: Springer-Verlag GmbH, 141 (2004) (cond-mat/0407622).

[13] D. Stauffer and P.M.C. de Oliveira, Eur. Phys. J. B 30, 587 (2002); D. Stauffer, Int. J. Mod. Phys. C 13, 975 (2002).

[14] A.A. Moreira, J.S. Andrade Jr. and D. Stauffer, Int. J. Mod. Phys. C 12, 39 (2001).

[15] R. Ochrombel, Int. J. Mod. Phys. C 12, 1091 (2001); F. Slanina and H. Lavicka, Eur. Phys. J. B, 35, 279 (2003).

[16] J. Bonnekoh, Int. J. Mod. Phys. C 12, 39 (2003).

[17] I. Chang, Int. J. Mod. Phys. C 12, 1509 (2001).

[18] M.C. González, A.O. Sousa and H.J. Herrmann, Int. J. Mod. Phys. C 15, 45 (2004).

[19] A.O. Sousa, Physica A 348, 701, (2005).

[20] L. Behera and F. Schweitzer, Int. J. Mod. Phys. C 14, 1331 (2004).

[21] J.R. Sanchez cond-mat/0408518.

[22] L.A.N. Amaral, A.Scala, M.Barthélémy and H.E. Stanley, Proc. Natl. Acad. Sci. U.S.A. 97, 49 (2000); F. Liljeros, C.R. Edling, L.A.N. Amaral, H.E. Stanley and Y.Aberg, Nature 411, 907 (2001).

[23] D.J. Watts and S.H. Strogatz, Nature 393, 440 (1998).

[24] P. Erdős and A. Rényi, Publicationes Mathematicae 6, 290 (1959).

[25] A.-L. Barabási and R. Albert, Science 286, 509 (1999); R. Albert and A.-L. Barabási Rev. of Mod. Phys. 74, 47 (2002); A.L. Barabási, Linked: The New Science of Networks, Perseus Publishing, Cambridge MA, 2002.

[26] J. Davidsen, H. Ebel and S. Bornholdt, Phys.Rev.Letters 88, 128701 (2002); P. Holme and B.J. Kim, Phys. Rev. E 65, 026107 (2002); G. Szabó, M. Alava and J. Kertész, Phys. Rev. E 67, 056102 (2003).

[27] A. Barrat and M. Weigt, Eur. Phys. J. B 13, 547 (2000). 